\title{
EL DERECHO \\ A LA PROTECCIÓN DE LA SALUD
}

Vicente Navarro

The Johns Hopkins University

\section{INTRODUCCIÓN}

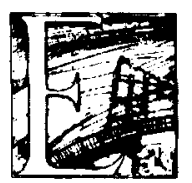

N 1974, el gobierno británico nombró una comisión de estudios de las desigualdades sociales en salud en Gran Bretaña, presidida por sir Douglas Black, cuyo informe final tuvo un gran impacto en la Gran Bretaña así como en el mundo occidental desarrollado. Era la primera vez que un gobierno occidental documentaba de una manera detallada la evolución de las desigualdades sociales en salud entre su población. Pronto, otros gobiernos siguieron los pasos del gobierno británico y establecieron comisiones con objetivos similares a la Comisión Black. Por fin, a mediados de los años ochenta, y como respuesta a la inquietud generada por estos estudios, la oficina europea de la OMS definió como objetivo prioritario de la política sanitaria europea la disminución de las desigualdades sociales en salud dentro de los países de la región europea. 
En España, el gobierno español (y concretamente su Ministerio de Sanidad y Consumo) estableció en octubre de 1994 una comisión de expertos con el objetivo de estudiar las desigualdades sociales en salud en España, dándole la responsabilidad de documentar y analizar aquellas desigualdades, con la invitación de realizar recomendaciones de cómo mejorar la salud de los españoles a través de políticas públicas que disminuyan las desigualdades sociales en salud en España. El objetivo explícito de la comisión que tuve el honor de presidir, era realizar un estudio semejante al que en su día realizó la Comisión Black en la Gran Bretaña. El día 7 de marzo de 1996, la Comisión presentó su informe final al gobierno español.

El intento de mi presentación es compartir con ustedes unas reflexiones personales sobre el desarrollo del derecho a la salud en España, que escribo a partir de la experiencia acumulada como presidente de aquella Comisión.

Quisiera subrayar que hago estas reflexiones a título personal y no como presidente de aquella Comisión. Ello me permite mayor libertad de expresión y mayor relajación en mi presentación, con la esperanza de estimular un necesario y urgente debate sobre la realización de aquel derecho (tan bien definido en la Declaración de los Derechos Humanos de las Naciones Unidas, escrita ya, en 1948, al final de la II Guerra Mundial) en España.

\section{EL DERECHO A LA ATENCIÓN SANITARIA}

Comencemos por aclarar que en gran número de casos (y en la gran mayoría de documentos escritos sobre la realización de tal derecho), éste se limita primordialmente al derecho de acceso y atención a los servicios sanitarios. Ésta es una visión de tal derecho excesivamente reducionista puesto que, como mostraré posteriormente, la salud de una población depende de factores mucho más importantes que la atención sanitaria. Pero, aceptando por el momento esta visión reducionista de tal derecho, los trabajos de la Comisión parecerían mostrar que hay una generalización y universalización de este derecho en España. El 98 por 100 de la población tiene cobertura sanitaria de financiación pública en España a partir del desarrollo de la Ley General de Sanidad que en la práctica universaliza esta atención.

No hay duda de que ello significa un gran logro y conquista social. Es más, en estudios realizados por la Comisión muestran que no existen desigualdades sociales en la utilización de servicios sanitarios. Una persona humilde utiliza los servicios sanitarios con la misma frecuencia que una persona de 
DERECHOS $Y$ LIBERTADES

mayores medios. La universalización de los servicios sanitarios ha permitido a amplios sectores de la población (gente humilde sobre todo y poblaciones rurales también) tener acceso y ser atendidos por los servicios que antes no les eran accesibles. Puesto que gran número de estas personas que estaban excluidas antes y que pueden ahora utilizar el sistema eran personas ancianas (que consumen los servicios sanitarios en mayor proporción que los adultos y jovenes) y de escasos medios, esta universalización de los servicios sanitarios ha tenido también un impacto muy importante en la redistribución de la renta en España, al permitir un mayor consumo de recursos por los grupos más humildes (y vulnerables). Es importante subrayar, por cierto, que la atención sanitaria pública tiene un efecto redistribuidor y equilibrador más fuerte que las transferencias sociales y la educación.

Otro resultado importante de la Comisión es que en aquellas actividades del sector sanitario cuyos servicios no se han universalizado y cuya financiación es predominantemente privada (como es el caso de los servicios odontologicos), las desigualdades sociales en la utilización de estos servicios continúa siendo muy marcada, y lo que es incluso más preocupante, estas desigualdades continúan acentuándose en lugar de reducirse. Esta realidad, bien documentada en los estudios de la Comisión, debiera estimular una reflexión en aquellas fuerzas que están pidiendo la privatización de la financiación de la sanidad en España. Los estudios de la Comisión son muy concluyentes. Tal privatización significaría la introducción y reproducción de desigualdades sociales en la utilización de los servicios sanitarios, con máximo perjuicio para los grupos más vulnerables, tales como está hoy ocurriendo en España con los servicios odontológicos de financiación predominantemente privada. La financiación pública y universalización del derecho a la atención sanitaria han permitido la eliminación de desigualdades sociales en la utilización de servicios. Ahora bien, esta realidad, documentada por la Comisión, requiere tres comentarios y observaciones.

Una de ellas es que el hecho de que el trabajador en paro, por ejemplo, tenga la misma utilización que el profesional liberal ocupado, no es necesariamente un indicador de que el derecho a la atención sanitaria se haya realizado en España. En realidad, necesidad e igualdad son dos conceptos distintos. Las encuestas de la Comisión muestran cómo el primero, el trabajador parado, se siente más enfermo y tiene más enfermedades que el segundo, el profesional ocupado, y por lo tanto, tiene mayores necesidades de atención sanitaria que el segundo. Si la utilización de servicios sanitarios, sin embargo, es la misma en los dos casos, ello quiere decir que en realidad (y conforme a la necesidad a 
aquella atención) el trabajador está subatendido pues debiera tener mayor y no igual utilización de servicios. Hay ahí un factor de subutilización que debiera preocuparnos.

Otra observación que creo importante subrayar es que el derecho a la atención sanitaria no se realiza cuando esta atención varía considerablemente según la región, residencia, e incluso clase social y tipo de ocupación, tal como la Comisión también documenta. No es lo mismo ser atendido en un ambulatorio (con un promedio de visita de entre uno y dos minutos) que en un nuevo centro de atención primaria (de tiempo de consulta promedio de entre cuatro y seis minutos) o en un despacho privado (de tiempo de consulta promedio de veinte minutos). Todas estas son varias formas de atención que se reciben por pacientes del SNS, dependiendo del lugar donde viven y del tipo de trabajo que realizan. Un catalán tiene menos acceso a los nuevos centros de salud y más a los ambulatorios antiguos que un andaluz o madrileño. Un funcionario público (administrador del Estado) tiene la libertad de escoger la medicina privada que se le niega a la mayoría de la ciudadanía. Estos son ejemplos de la gran variabilidad en el desarrollo de tal derecho, variabilidad que se presenta incluso entre aquellos ciudadanos y residentes que tienen su cobertura sanitaria financiada públicamente. Vemos así una gran diversidad de servicios y beneficios, dentro del SNS que diluye el principio de que todo ciudadano y residente debiera tener los mismos derechos de acceso y beneficios y que éstos se distribuyeran sólo conforme a las necesidades de la población. Esta dimensión de los derechos a la atención sanitaria no está desarrollada ni garantizada en España. Las variaciones y desigualdades en la atención sanitaria son todavía hoy muy notables.

Aquí valga hacer otra observación que creo tiene también relevancia en cuanto a las posibilidades de que este desigual desarrollo del derecho de acceso y utilización de los servicios sanitarios se corrija. En España, la mayoría de los profesionales y políticos que deciden y ejecutan las decisiones sanitarias utilizan la atención primaria privada. Los resultados de los estudios y encuestas realizadas en España muestran que tanto los miembros de las Cortes como los funcionarios del Ministerio de Sanidad utilizan el sistema MUFACE que (aunque financiado públicamente) les permite utilizar la medicina privada. Estos dirigentes y administradores no conocen ni sienten la realidad experimentada por la mayoría de la ciudadanía, distanciándose así la experiencia del gobernante de la del gobernado. Una situación semejante ocurre en otros países y sectores, lo que ha popularizado en países como Estados Unidos medidas adoptadas por referéndum populares (a nivel de algunos Estados), condicionando el permiso para que a una persona se la acepte como candidato para un puesto político a 
que se comprometa (él/ella y su familia) a utilizar los servicios públicos (servicios sanitarios y escuelas primarias y secundarias) utilizados por la mayoría de la población. Creo que sería aconsejable que una medida semejante se considerara en España aunque tengo que admitir cierto pesimismo a que se aprobara. La atención primaria a nivel de los ambulatorios es muy insuficiente y ello responde a realidades políticas más que a condiciones económicas.

La asistencia sanitaria, sin embargo, no es la causa mayor del nivel de salud de una población. La evidencia científica que apoya esta afirmación es muy abundante. Ello no implica que tal asistencia no tenga valor o importancia. Antes al contrario, estos servicios sanitarios son importantes, no tanto en curar enfermedades sino en tomar cuidado de ellas y de las personas enfermas. En España, como en todos los países desarrollados, la mayoría de enfermos son enfermos crónicos. La morbilidad prevalente en países avanzados es una morbilidad crónica. La estrategia sanitaria dominante debiera ser por lo tanto la estrategia de tomar cuidado de más que la de curar. Naturalmente que no estoy desmereciendo la importante función de curar (en la medida que el sector sanitario pueda curar), sino subrayando que al menos numéricamente los problemas más importantes que tiene la medicina española es la de prevención y tomar cuidado de la morbilidad crónica. Y ahí está claro que la sanidad española no está suficientemente preparada para ello. Valga únicamente subrayar el enorme subdesarrollo de los servicios médico-sociales comunitarios de atención a los ancianos e incapacitados. Esta atención, en España, la realizan las hijas de los ancianos, a un coste personal elevado. Las mujeres del grupo 3555 tiene muchas más enfermedades relacionadas con el estrés que cualquier otro grupo etario femenino o masculino en España. Las grandes deficiencias del estado de bienestar español las está cubriendo la mujer española a un coste personal muy elevado. Los estudios de la Comisión muestran esta realidad.

Otra dimensión de la estrategia sanitaria de tomar cuidado es la importancia que la satisfacción del usuario debiera tener como indicador de la calidad del sector sanitario. Este punto es muy importante que se subraye, puesto que en España el criterio más extendido de evaluación de los servicios sanitarios es valorar su impacto en la mortalidad y morbilidad (es decir en el número de muertos y enfermedades), indicadores que creo insuficientes, e incluso erróneos, puesto que muchos otros factores, incluso más importantes que los servicios sanitarios, están determinando el nivel de mortalidad y morbilidad en España. En cambio sí que creo que un indicador muy válido para evaluar la atención sanitaria es el grado de satisfacción que el usuario tiene de la atención que recibe, un criterio de evaluación poco extendido en el sector sanitario donde el 
usuario no ocupa la centralidad que debiera. En este aspecto es interesante subrayar que la clase trabajadora y clases populares están más satisfechas con el SNS (y muy en especial con la atención sanitaria) que las clases medias. Ello se debe al distinto nivel de expectativas, más altas entre las clases medias que entre las clases populares. En realidad, podemos ver también en los trabajos de la Comisión que las clases medias y altas utilizan los servicios sanitarios privados (y muy en particular los servicios de atención primaria privados) en porcentajes mucho más altos que las clases populares. Las encuestas muestran también que el porcentaje de la población insatisfecha con el servicio público es más alto entre las clases medias y altas que entre las clases populares. Esta situación es preocupante porque debilita al estado de bienestar. En la Europa de tradición democrática, el estado de bienestar ha sido resultado de la alianza de la clase trabajadora con las clases medias. Esta alianza fue el motor de expansión del estado de bienestar. Si las clases medias se distancian del estado de bienestar, éste se empobrecerá, al disminuir la presión por su mejora. De ahí que el objetivo de aquella alianza fue siempre la universalización de los beneficios y servicios del estado de bienestar procurando que el nivel de calidad de los beneficios y servicios satisficieran incluso a los grupos y clases más exigentes. Y este es el reto del SNS. Lograr que las clases medias se encuentren satisfechas con los servicios públicos. Es importante reconocer que entre los usuarios (a diferencia de entre la población en general), y muy en particular, entre los usuarios de los servicios hospitalarios, la distancia entre los niveles de satisfacción de las clases medias y de las clases trabajadoras disminuye. Pero persiste entre los usuarios un gradiente de satisfacción e insatisfacción dependiendo de la clase social y del nivel de renta, lo cual señala que hay todavía mucho por hacer en el SNS, sobre todo en mejorar el trato personal, respetuoso y amistoso ( $y$ no condescendiente) hacia el usuario y familiares, la capacidad de elección de profesionales sanitarios e instituciones, y los aspectos hoteleros de la atención. Estos son los retos más importantes de la sanidad española en su dedicación a la plena realización de los derechos a la atención sanitaria de la población española.

\section{DERECHO A LA SALUD COMO DERECHO DISTINTO AL DERECHO DE ATENCIÓN SANITARIA}

Hasta ahora he hablado del derecho a la atención sanitaria como un componente importante pero limitado e insuficiente del derecho a la salud. Como he indicado ya en varias ocasiones en esta conferencia, la salud de 
cualquier población depende mucho más de intervenciones económicas sociales y políticas que de las intervenciones sanitarias. Y la oficina de la OMS lo explicita bien claro cuando habla de que la salud de la población depende en gran manera de la cantidad y calidad del trabajo, de la vivienda, de la alimentación, de la educación y del bienestar social dentro de un ambiente de paz y desarrollo social. Y estas condiciones están hoy distribuidas de forma desigual en España. La Comisión ha mostrado que mientras han desaparecido las desigualdades sociales en la utilización de los servicios sanitarios por clase social, así y todo las desigualdades en los niveles de salud continúan existiendo entre las clases sociales. Por todas las condiciones diagnósticas estudiadas (las causas mayores de mortalidad y morbilidad en España) hay un gradiente de mayor a menor según la clase social. La burguesía tiene menos enfermedades y mortalidad que la pequeña burguesía y ésta menos que las clases medias y éstas menos que la clase trabajadora, y dentro de ésta, la clase trabajadora calificada menos que la no calificada, y dentro de cada clase, los ocupados tienen menos enfermedades y mortalidad que los parados, y los de mayor renta menos que los de menor renta. La única excepción a esta situación es la alergia por causas que desconocemos. Ahora bien, ésta es la excepción que confirma la regla.

De esta situación se derivan varias conclusiones. Una de ellas, pertinente a esta conferencia, es que la realización del derecho a la salud varía en España según la clase social, estado laboral y nivel de renta de los ciudadanos y residentes españoles.

La segunda conclusión es que la estrategia más eficaz en la reducción de mortalidad y morbilidad en España es precisamente la reducción de las desigualdades sociales en salud. Si todas las clases sociales tuvieran la misma mortalidad y morbilidad que la clase más pudiente, se prevendrían muchas más muertes y enfermedades que cualquier otro tipo de intervención sanitaria hoy contemplada en España a través del SNS. De ahí que la oficina europea de la OMS haya definido como uno de los objetivos más importantes y eficaces para reducir la mortalidad y morbilidad la reducción de las desigualdades sociales.

Otra tercera conclusión es que la estrategia de reducción de desigualdades sociales beneficia a toda la población y no sólo a los grupos vulnerables y/o marginados. Como he dicho anteriormente, existe un gradiente de mortalidad y morbilidad, por clase social, y dentro de cada clase social por nivel de renta y ocupación. De ahí que la reducción de desigualdades mejore la salud de todas las poblaciones afectadas por tal reducción. 
En este aspecto es importante subrayar algunos resultados de los trabajos realizados por la Comisión que han analizado la evolución de las desigualdades sociales en salud durante el período 1987-1993. Y los resultados son mixtos, es decir positivos en muchos aspectos, no tanto en otros. Entre los primeros, ha sido la disminución de la desigualdad en la valoración del estado de salud entre las clases sociales, y muy en especial entre los grupos etarios por encima de los cincuenta y cinco años. Sin embargo, esta disminución importante de las desigualdades sociales en salud se ha producido particularmente en las Comunidades Autónomas con renta familiar disponible igual o mayor a la renta media nacional. En las Comunidades Autónomas con renta familiar disponible significativamente inferior a la media nacional las desigualdades sociales en salud entre las clases sociales han aumentado. Es decir, la reducción de las desigualdades sociales en salud está siendo más exitosa en las Comunidades Autónomas ricas que en las pobres. Aquellas desigualdades continúan más persistentemente en las comunidades pobres que en las ricas. En realidad, la causa de su peor salud es precisamente la mayor persistencia de las desigualdades sociales en salud dentro de ellas. Este punto merece resaltarse puesto que este aspecto -las desigualdades sociales en salud dentro de cada Comunidad Autónoma - no ha sido discutido e incluso analizado con la misma intensidad que las diferencias de salud entre las Comunidades Autónomas.

\section{EL DERECHO A LA SALUD: LAS DESIGUALDADES ENTRE LAS REGIONES AUTÓNOMAS}

En cuanto a este aspecto, las desigualdades sociales en salud entre las Comunidades Autónomas, los estudios de la Comisión muestran que, en el caso de mortalidad, las Comunidades Autónomas del norte-noreste (de nivel de renta más alto) tienen una mortalidad menor que las Comunidades Autónomas del sur-sureste, confirmándose así la división en diagonal de España, entre la España próspera y la menos próspera. La Comisión también ha documentado que es en esta segunda España donde hay mayores porcentajes de la población que viven en zonas deprimidas, tienen mayor analfabetismo, y tienen mayores desigualdades sociales en salud. Dentro de ellas destacan Extremadura y Andalucía, con un mayor porcentaje de desempleados, con tasas de desempleo próxima al 30 por 100 . Este desempleo es de origen primordialmente agrícola. 
La Comisión también analizó el primer estudio ecológico de municipios (áreas menores que la provincia) en España, analizando las desigualdades sociales en salud a nivel de áreas pequeñas y a partir de regiones. Este estudio confirmó los otros estudios también realizados por la Comisión, que dividen a España en tres tipos de regiones. Unas situadas preferentemente en el nortenoreste de España, que incluye Comunidades Autónomas como La Rioja, Cantabria, Aragón, Navarra y Cataluña que tienen mejores indicadores de salud (y menos desigualdades sociales en salud), otra intermedia con niveles intermedios de salud (que incluye Comunidades Autónomas como Valencia y Galicia) y otra de niveles bajos de salud (como Andalucía, Extremadura y Canarias) con grandes desigualdades sociales en salud. En el análisis de estas regiones es importante repetir de nuevo lo dicho anteriormente, es decir, que para explicar el nivel de salud de una comunidad es tan importante analizar las desigualdades sociales entre como dentro de las Comunidades Autónomas, una realidad que raramente es constatada en los documentos oficiales sobre el estado de salud del pueblo español.

\section{DESIGUALDADES SOCIALES EN SALUD POR GÉNERO}

La Comisión también estudió las desigualdades sociales en salud por género y vio que, en cuanto a mortalidad, había mayor mortalidad entre los varones que entre las mujeres, sobre todo a partir de los sesenta y cinco años. De ahí que España tenga más ancianas que ancianos. Caso distinto ocurre con la morbilidad en que por regla general había mayor morbilidad entre las mujeres del grupo etario 35-55 años que cualquier otro grupo etario (excepto ancianos) y que los hombres, por las razones enunciadas anteriormente.

Las diferencias mayores, sin embargo, fueron en los hábitos personales donde las diferencias de comportamiento eran muy acentuados. En este aspecto, los resultados de los estudios de la Comisión son altamente preocupantes. Mientras que durante el período 1987-1993 hubo un descenso en la prevalencia del tabaquismo entre los hombres, ocurrio un aumento muy marcado entre las mujeres y sobre todo entre las mujeres jóvenes, grupo al que la industria tabacalera centra su promoción. Y ello ocurría en todas las clases sociales. Es más, mientras que la reducción del tabaquismo entre los hombres ocurría en todas las clases sociales (incluyendo las clases populares que son las que tienen mayor prevalencia de tabaquismo) el aumento del tabaquismo entre las 
mujeres ocurría en todas las clases sociales (y predominantemente entre las clases populares que habían tenido históricamente una prevalencia de tabaquismo menor que las clases altas y medias). La Comisión valoró esta situación muy negativamente.

El otro comportamiento estudiado por la Comisión fue el alcoholismo (bebida de alcohol en exceso) en el que había también un comportamiento distinto dependiendo no sólo de la clase social sino también del género. La prevalencia del alcoholismo es mucho mayor en los hombres que en las mujeres, y en las clases populares que en las clases medias y altas. En cambio la Comisión no encontró un patrón claro de diferenciación social por clase entre las mujeres, en alcoholismo.

Es importante señalar que la mayor prevalencia en general del comportamientos insanos en las clases populares que en las clases más pudientes, es más un síntoma que una causa de las desigualdades sociales en salud. Es decir que estos comportamientos se generan y reproducen en respuesta a unas bases materiales que son la causa de aquellas desigualdades. De ahí que la experiencia internacional en campañas de educación sanitaria muestra que la eficacia de estas compañías encaminadas a cambiar comportamientos es limitada cuando no se cambian las bases materiales (trabajo, vivienda, educación, etc.) que sustenten aquellos comportamientos. De ahí que, aunque tales campañas sean importantes (y es necesario que se promuevan muchas más campañas en España), todavía más importante y eficaces son las intervenciones encaminadas a reducir las desigualdades sociales. La evidencia científica en este aspecto es muy convincente. Esta evidencia muestra no sólo que las diferencias sociales de mortalidad son mucho mayores que las diferencias de comportamiento, sino también que, como decía antes, estas diferencias de comportamiento responden a diferencias sociales. En realidad las explicaciones del porqué de las desigualdades sociales en salud no pueden ser monocausales. No es la variable comportamiento, o renta, o nivel de educación, o tipo de trabajo, o cualquier otra variable la que en sí explica las desigualdades sociales. De ahí que intervenciones univariantes sean insuficientes. Es la relación (que es distinto a la suma) entre cada una de estas variables, dentro de una matriz de relaciones sociales, como son las clases sociales, que determinan las diferencias en salud. Éste es, en realidad, uno de los temas que hoy acaparan más la atención de los estudiosos de las desigualdades sociales en salud. Como se preguntaba recientemente una editorial del New England Journal of Medicine (vol. 223, 1995), «que hay en la estructura y relaciones de clase que expliquen las desigualdades sociales en salud». «¿Por qué -continuaba preguntando aquella editorial- un trabajador no calificado tiene mayor mortali- 
dad y morbilidad que un profesional liberal?» La respuesta no es sólo que uno tiene más recursos y consume más que el otro. Es mucho más que eso. El primero carece, por lo general, de un apoyo e infraestructura de apoyo social, tiene poca estima de sí mismo, tiene gran inseguridad y estrés, un trabajo repetitivo, con escasa exigencia de creatividad, con escaso control sobre su ambiente laboral, con una individualización y atomización de su trabajo, y en una situación represiva de constante supervisión. Estos factores psicosociales determinan una personalidad y comportamientos, y una manera de enfermar y morir. Como escribía recientemente el profesor Wilkinson, estos factores en sí pueden ya explicar el 90 por 100 de las mortalidades diferenciales entre las clases sociales ${ }^{1}$.

Es también importante subrayar el componente relacional en las desigualdades sociales como causa de las desigualdades sociales en salud. Desigualdad es un concepto relacional y viene definido por las relaciones sociales. La pobreza, por ejemplo, no es un concepto absoluto sino relacionar. Un pobre en Harlem, New York, por ejemplo, tiene mayores recursos materiales ( 8.000 dólares por año) que una persona de clase media en Zambia. En términos absolutos, parecería entonces que el pobre de Harlem debiera tener mejores indicadores de salud (como por ejemplo menos mortalidad) que la persona de clase media de Zambia. Pero en cambio no la tiene. En realidad, el pobre de Harlem tiene peores indicadores y ello debido a que ser pobre en Harlem es mucho más difícil (e insano) que ser una persona de clase media en Zambia. El primero sufre más su pobreza, está más marginado, tiene menos respeto de sí mismo, está en una situación más vulnerable, que el segundo. Lo que enferma al pobre de Harlem es la distancia que le separa de lo promedio y de lo que es definido como exitoso. De ahí, la enorme importancia de analizar las desigualdades sociales dentro de cada país analizando la base material, la estructura y arquitectura social que genera y reproduce estas desigualdades, como medida más adecuada para entender las desigualdades sociales en salud.

Naturalmente que esta estructura social, esta fábrica social dentro de cada sociedad está afectada por la desigualdad social a nivel internacional y global. Estamos hoy viendo, por ejemplo, un aumento muy notable de las desigualdades sociales a nivel internacional que están favoreciendo y estimulando a su vez un incremento de las desigualdades sociales a nivel de cada país y ello en respuesta a unas políticas económicas que están teniendo efectos negativos en los niveles de salud, incluso de los países desarrollados. En Estados Unidos, por

1 WILKINSON, R., «Health, Distribution and Growth», en Glyn, A. y Miliband, D., Paying for Inequality. The Economic cost of Social Injustice, River Draw Press, London, 1995, p. 36. 
ejemplo, el aumento muy notable de las desigualdades sociales estimulado por aquellas políticas han creado una polarización social sin precedentes que ha causado que por primera vez desde hace quince años haya habido un aumento de la mortalidad y descenso de la esperanza de vida al nacer en aquel país. De ahí que sea tan importante que se evalúen y sigan los costos sociales de aquellas políticas a fin de determinar su impacto en la calidad de vida de la población y en la realización de sus derechos a la salud.

La relación entre lo local, regional, nacional e internacional se ve más y más entrelazado. Y es difícil resolver las problemáticas locales con intervenciones meramente locales. Por ejemplo, la diferencia de esperanza de vida entre los barceloneses del barrio de Montjuic y los del barrio de Pedralbes es de diez años. La burguesía y pequeña burguesía en Barcelona viven diez años más que sectores de las clases de bajos ingresos y marginados y ello a pesar de las políticas correctoras de las desigualdades sociales en salud del Ayuntamiento de Barcelona, que la Comisión definió como ejemplares. Pero estas políticas debieran complementarse con políticas regionales, nacionales e incluso internacionales que permitieran estas reducciones. Este es el gran reto de los defensores de la realización de los derechos humanos. El articular una respuesta a todos estos niveles. Una labor inmensa pero quizás realizable. Así lo espero.

(Véase el Capítulo 7. Conclusiones y Recomendaciones, del Informe de la Comisión Científica sobre Desigualdades Sociales en Salud en España del Ministerio de Sanidad y Consumo del Gobierno español.)

\section{Conclusiones y recomendaciones (escritas y hechas por la Presidencia de la Comisión)}

Conclusiones y recomendaciones de la Comisión de Estudios de las Desigualdades Sociales en Salud en España siguiente:

De los estudios y seis capítulos de la Comisión se concluye y recomienda lo

\section{Captrulo I}

I.1 La salud de la población humana a nivel mundial (y muy en particular el descenso de la mortalidad) ha mejorado de una manera espectacular durante el siglo $\mathrm{xx}$, con un aumento de la esperanza de vida durante este siglo mayor que el aumento experimentado por la población humana durante todo el período transcurrido desde el siglo I al siglo XIX. Esta mejora ha sido causada primordialmente por el gran des- 
arrollo económico y social ocurrido durante estos últimos cien años, junto con el impacto de políticas redistributivas que han contribuido a la reducción de las desigualdades sociales existentes a nivel mundial, permitiendo así el acceso de amplios sectores de la población a recursos vitales de los que carecían. Entre estos recursos cabe señalar las intervenciones de saneamiento del medio ambiente, las medidas de salud pública ,y en menor grado, los avances en la eficacia de los servicios médicos sanitarios y la ampliación de la cobertura sanitaria a poblaciones previamente excluidas del acceso a estos progresos. El informe detalla cómo la mejora de las condiciones de salud y bienestar social de la población mundial se deben a causas económicas, sociales y políticas más que a las intervenciones salubristas o incluso sanitarias.

Basándose en esta conclusión, recomendamos que el gobierno español enfatice en los foros internacionales la necesidad de estudiar no sólo el impacto que el crecimiento de la población tiene en el desarrollo económico y social de las sociedades, sino también el impacto que los factores sociales, económicos y políticos están teniendo en el nivel de salud y bienestar social de las poblaciones. Creemos que mientras que la comunidad internacional ha patrocinado varias conferencias y foros sobre el impacto del crecimiento de la población en el bienestar social del mundo, no ha puesto la misma atención (excepto en la última Conferencia Internacional de Copenhague sobre Desarrollo Humano) en el impacto que las fuerzas económicas, sociales y políticas están teniendo en la salud y bienestar social de las mismas poblaciones.

I.2 La espectacular mejora de la salud y bienestar social de la población mundial durante este siglo no puede ser motivo de complacencia puesto que queda mucho por hacer. Todavía hoy mueren 14 millones de niños al año debido al hambre, equivalente, por lo tanto, a la mortalidad causada por bombas atómicas como las que explotaron en Hiroshima y Nagasaki hace cincuenta años, bombas de hambre que continúan explotando año tras año, sin producir ningún ruido, y sin que por lo visto merezcan aparecer en los medios de información del mundo desarrollado que consideran el hecho de que un niño muera de hambre cada dos segundos, como parte de una realidad aceptada como inevitable en nuestra civilización. La Comisión señala que la gran mayoría de estas muertes son fácilmente prevenibles desde el punto de vista científico y salubrista. El hambre, el problema de salud pública más importante hoy en el mundo, es de fácil resolución técnica, puesto que hoy existen en el mundo suficientes alimentos para prevenir estas muertes. Es más, los gobiernos están subvencionando a los productores agrícolas en los países subdesarrollados para que no produzcan más alimentos. Que esta situación se reproduzca responde a los mismos factores políticos, económicos y sociales que mantienen e imponen las desigualdades sociales en la distribución de recursos de la población.

Además de la importancia que atribuimos a la concienciación de lo mucho que queda por hacer, la Presidencia de la Comisión también alerta del riesgo de inversión de las mejoras tan notables experimentadas en el nivel de salud y bienestar social durante el siglo $\mathrm{XX}$, con el deterioro de ambos en algunos países, tanto entre los países con grandes recursos como entre quienes tienen menos recursos. Ello se debe a los cambios de la estructura económica y social mundial y a políticas públicas que están invistiendo y exacerbando las desigualdades sociales a nivel mundial. El informe muestra cómo está disminuyendo con rapidez la mejora del estado de salud y bienestar en muchos países e incluso cómo se deteriora en otros muchos, debido las causas mencionadas. 
Por ello, recomendamos que el gobierno español sugiera a la comunidad internacional que:

a) Se desarrollen sistemas de información nacionales e internacionales que permitan seguir la evolución de la desigualdad social a nivel mundial, así como sus consecuencias en el estado de bienestar social y salud de la población mundial.

b) Se establezcan criterios de valoración y evaluación de políticas económicas $Y$ sociales según el impacto que éstas tengan en las desigualdades sociales y en la salud y bienestar social de las poblaciones. Evaluar las políticas económicas según su impacto en la tasa de crecimiento del PIB per cápita es insuficiente para explicar el nivel de salud de las poblaciones, puesto que se ignora el elemento más importante para explicar el nivel de salud y bienestar social, a saber, su impacto en las desigualdades sociales.

I.3 Consideramos que las desigualdades sociales son un obstáculo para mejorar el nivel de salud y bienestar social de la población, e incluso el crecimiento economico. De ahí que coincidamos con el diagnóstico del Banco Mundial de que «no existe evidencia de que el ahorro dependa de la desigualdad social o que ésta, la desigualdad social, conduzca a un mayor crecimiento económico. En realidad, la evidencia apunta a un sentido contrario, es decir, que mayor desigualdad lleva a menor crecimiento económico» (BM, 1991).

I.4 Las reducciones de las desigualdades sociales beneficien al nivel de salud de toda la población. El informe de la Comisión muestra con gran detalle la existencia de un gradiente en el nivel de salud y estado de bienestar en la población, en el que las clases más beneficiadas tienen mejores indicadores de salud y bienestar social que las clases con menos recursos. Es más, dentro de cada clase, los individuos con mayores recursos tienen en general mejores indicadores de salud que aquellos individuos que tienen menos recursos. De ahí que las políticas públicas encaminadas a reducir las desigualdades sociales mejoren la salud y bienestar social de toda la población y no sólo la de los grupos más vulnerables. Mas aun, las políticas sanitarias más eficaces para mejorar los niveles de salud de la población, son aquellas que reducen las desigualdades sociales en salud. Por ejemplo, si hoy en España, las clases IV y V (clases trabajadoras manuales) tuvieran la misma mortalidad que las clases I (profesionales y directivos) se prevendrían un número de muertes mucho mayor que cualquier otra medida salubrista hoy considerada dentro de las estrategias sanitarias. De ahí que recomendamos:

a) Que el gobierno español considere también como prioritario el objetivo marcado por la Oficina Europea de la OMS de reducir las desigualdades sociales como uno de los mejores medios de mejorar la salud de las poblaciones y que lo realice y desarrolle como parte de su política sanitaria, estimulando y facilitando la extensión de este objetivo a los gobiernos autonómicos y municipales.

b) Que las politicas sanitarias y sociales sean universales y afecten a la mayoría de la población y no sólo a los grupos vulnerables. En este aspecto queremos distanciarnos de la visión que considera como políticas sociales a las exclusivamente a políticas que las poblaciones están dirigidas vulnerables o con necesidades específicas. Entendemos las políticas sociales como aquellas que mejoran la condición social de 
toda la población, puesto que toda persona que está en una situación desigual puede ver su bienestar social y su salud mejorada. Toda la población está en situación de riesgo debido a las desigualdades.

1.5 En España la Comisión documenta los logros que se han alcanzado, sobre todo con los gobiernos democráticos, en la mejora de las desigualdades sociales en salud. Las fuerzas democráticas del Estado español merecen el aplauso de la Comisión por su compromiso en reducir desigualdades muy acentuadas, heredadas de regímenes anteriores con escasa sensibilidad social. Ahora bien, la Comisión también documenta que todavía hay mucho por hacer y que incluso, en algunos casos, existe un aumento de las desigualdades sociales en salud. De ahí que recomendamos que:

a) Los distintos niveles de gobierno existentes en España desarrollen los sistemas de información recomendados en capítulos posteriores para monitorizar la evolución de las desigualdades sociales en salud.

b) Que el Gobierno y el Parlamento español establezcan una comisión permanente que analice la evolución de las desigualdades sociales en salud en España y que les asesore sobre la manera en cómo disminuir aquellas desigualdades.

c) Que las autoridades sanitarias tengan mayor incidencia en el desarrollo de políticas económicas y sociales que puedan afectar negativamente a las desigualdades sociales en salud, previniendo o disminuyendo tal efecto.

d) Que las autoridades sanitarias y de servicios sociales coordinen sus intervenciones entre sí, así como con las autoridades económicas y agentes sociales de la sociedad civil a fin de incidir sobre las desigualdades sociales en salud.

e) Que los programas de formación e investigación de los profesionales sanitarios incluyan, con mayor detalle y extensión en sus programas de formación, el estudio de las causas de producción y reproducción de las desigualdades sociales en salud, ampliando el enfoque excesivamente biologicista que todavia hoy domina en la enseñanza de aquellos sectores.

\section{CaptTulo II}

II.1 La información empírica presentada en el informe evidencia que las correcciones de las desigualdades sociales en salud son una de las estrategias más importantes para mejorar la salud y el estado de bienestar social de la población española. La Comisión concluye, por lo tanto, que es necesario y urgente documentar el estado de las desigualdades sociales en salud e incidir para reducirlas. Una condición para ello es una mejor elaboración conceptual, y una mejor medición de las desigualdades sociales en salud en España mediante una mejora sustancial del sistema de información existente.

De ahí que recomendamos que en los principales sistemas de información sanitarios y sociales se recoja información sobre la situación social de los españoles y no sólo de sus condiciones biológicas (talen como el sexo y la edad) únicas variables (además del lugar de residencia) que se recogen consistentemente en estos sistemas de información. 
II.2 Las desigualdades sociales en salud tienen varias dimensiones, entre las que se incluyen las desigualdades por clase social, género, edad, región geográfica, estado laboral y civil, y localización urbana y rural.

De ahí que recomendamos que la información sobre estas distintas dimensiones de las desigualdades sociales en salud debiera recogerse en:

a) Las encuestas de salud, tanto a nivel del Estado como en las encuestas de las Autonomías o las ciudades.

b) Los registros de enfermedad.

c) Los registros de mortalidad, incluyendo el boletín estadístico de defunción.

d) Los registros de mortalidad, incluyendo el boletín estadístico de natalidad.

e) Las historias clínicas, y

f) El conjunto mínimo básico de datos

II.3 Aunque los registros y sistemas de información mencionados anteriormente recogen información sobre la edad, el género, el lugar de residencia, y el estado civil, muy pocos recogen información sobre la clase social y estado laboral de los ciudadanos y residentes, variables que son de enorme importancia para el conocimiento de las desigualdades sociales en salud en España. La evidencia empírica presentada en este informe y en la literatura científica nacional e internacional muestran que las desigualdades sociales en salud por clase social se hallan entre las más importantes, persistentes y continuadas tanto en España como en otros países europeos. La falta de información sobre esta variable crea un desconocimiento muy grave de una de las causas mayores de mortalidad, morbilidad, enfermedad, salud y bienestar social, es decir, las desigualdades por clase social.

Las variables más frecuentemente utilizadas para definir la clase social de las personas han sido la ocupación, la educación y el nivel de renta. La Comisión ha analizado las ventajas y desventajas de cada una de ellas y su Presidencia recomienda que:

a) La ocupación como la más importante y pertinente, aunque señala la necesidad de utilizar otros indicadores conjunta o separadamente con la ocupación.

b) Utilizar las ocupaciones y grupos de ocupaciones recomendadas por la Sociedad Española de Epidemiología (SEE, 1995), o variaciones de ellas, tal como hemos realizado en este informe. La Comisión cree que para definir la clase social y para entender las desigualdades sociales en salud por clase social hay maneras más elaboradas y más adecuadas que la tipología utilizada en este informe, o la recomendada por la Sociedad Española de Epidemiología. Sin embargo, la infraestructura de recolección de datos y sistemas de información española no permite la puesta en marcha de tales clasificaciones.

Recomendamos que en una deseable ampliación de los sistemas de información sobre desigualdades sociales en salud se incluyeran también otros tipos de información social, tales como el tipo de control, autoridad, y supervisión existente en la ocupación, el tipo de empleo (pleno o parcial), las fuentes de ingresos y propiedad, y el tamaño de la familia, además del tipo de vivienda y el grado de cualificación. Estas son las variables recomendadas para las encuestas de salud y registros civiles e historias clínicas por el grupo de trabajo «Measuring Social Inequalities in Health», patrocinado por el gobierno federal de Estados Unidos, que también recomendó que los registros y encues- 
tas permitieran la conexión entre bases de datos («linkage»), siempre y cuando se mantuviera la confidencialidad. La Comisión concuerda también con esta recomendación.

II.4 Las tipologías de clase social utilizadas en gran parte de la literatura científica española en temas de desigualdades sociales carecen de una elaboración teórica adecuada que permita analizar la relación existente entre los distintos componentes y dimensiones de clase social y salud. Por regla general, estas tipologías siguen una concepción jerárquica de la sociedad, con un gradiente de autoridad o competencias, que no responde a criterios científicos. Por ejemplo, dividir la sociedad en clases altas, medias y bajas no sólo es peyorativo para las clases populares (definidas como clases bajas) sino que constituye una práctica ideológica. Supone un orden jerárquico en el que los grupos sociales con mayores recursos se definen como altos dentro de un orden jerárquico social. Es más, el método de preguntar a la población por su autoidentificación de clase, invitando a la población a que se defina como clase alta, media o baja, predispone a tener respuestas muy sesgadas puesto que la evidencia muestra que la población prefiere autodefinirse como clase media en lugar de como clase baja, exagerando el porcentaje de la población que se considera de clase media. Por otra parte, la evidencia también muestra que si a la población se le pide que se autodefina como miembros de la burguesía, pequeña burguesía, clase media y clase trabajadora (la mayoría se define trabajadora) los resultados son muy distintos. De ahí que recomendemos que:

a) Las clases sociales se definan bien por las categorias basadas en el modelo Golthorpe o, en el caso de que se pida la autodefinición de pertenencia a una clase, se utilicen los términos burguesía, pequeña burguesía, clase media y clase trabajadora, términos que además de ser más científicos son más afines al discurso y cultura popular que los utilizados como clases altas, medias o bajas. Los términos burguesía, pequeña burguesía, clases medias y clase trabajadora son términos científicos, ampliamente utilizados por distintas tradiciones sociológicas y económicas en el estudio de estructuras sociales.

b) Tanto las encuestas nacionales de salud como otras encuestas de ámbito local cambien la utilización de los términos clases altas, medias y bajas para la autodefinición de la población, evitando reproducir asi un esquema ideológico que mantiene unas jerarquías sociales que mantienen valoraciones contrarias al intento de disminuir las desigualdades sociales. Les recomendamos que utilicen en su lugar los términos de burguesía, pequeña burguesia, clase media y clase trabajadora que tienen un contexto científico más riguroso y una aceptación popular más adecuada.

II.5 Que la ocupación continúe constando en el certificado de defunción, y que en el caso de los jubilados se ponga la ocupación tenida antes de su jubilación. Que esta información se rellene por el médico, o el familiar del difunto, o la agencia funeraria como parte del registro civil.

II.6 Que la formación de profesionales sanitarios se enriquezca con la dimensión social, además de la biológica, del conocimiento médico y sanitario, sensibilizando a los profesionales de la importancia de recoger datos sociales en su práctica sanitaria. En este aspecto, exponemos nuestra profunda preocupación por el profundo biologicismo del conocimiento médico y su escasa sensibilidad por el contexto social de la salud y de la enfermedad. 
II.7 A fin de conocer la evolución de las desigualdades en salud es fundamental que las encuestas nacionales de salud utilicen las mismas variables sociales, conserven las mismas preguntas, y se realicen en las mismas fechas a fin de permitir la comparabilidad de los datos a través del tiempo.

\section{Capítulo III}

III.1 La percepción de que los grupos sociales enferman, mueren y reciben una atención sanitaria desigual, posee una larga tradición histórica aunque la información disponible es muy desigual entre los distintos países.

III.2. A principios de los años ochenta, la publicación en Gran Bretaña del informe conocido como "Black Report» constituy6, por su impacto social inmediato y por su influencia posterior, un hito de primera magnitud, un punto de inflexión en la historia moderna del estudio de las desigualdades en salud, que estimuló la creación de comisiones de estudio para analizar estas desigualdades. Su principal conclusión fue: tras treinta años de existencia del Servicio Nacional de Salud Británico, las desigualdades sociales en salud y en el uso de los servicios sanitarios y preventivos por clase social son claras y, lo que es más preocupante, están aumentando.

III.3 Según el informe «The Health Divide» que continuó la tradición del informe Black, las desigualdades en salud en Gran Bretaña persistieron o se ampliaron desde 1980; las recomendaciones propuestas en el «Black Report» prácticamente no recibieron respuesta. El propio informe confirmó las conclusiones del informe Black: «Desde 1950, las desigualdades en salud para los adultos en edad de trabajar se han ampliado. La excepción a esa tendencia se encuentra en los niños de un mes a un año de edad.» Las clases más desaventajadas tienen tasas de consulta médica más elevadas, pero usan menos los servicios preventivos. Además, se observan desigualdades regionales muy claras entre el norte y el sur del país. Aunque se encuentran áreas pobres en todas las regiones, éstas son mucho más comunes en el norte que en el sur o en el sur-este. No se han adoptado políticas para reducir la pobreza infantil, o mejorar las vivienda, y sólo en muy pocas ocasiones se han adoptado mecanismos para coordinar las políticas sanitarias a nivel nacional y local.

III.4 Actualmente, el estado de la investigación sobre desigualdades en salud difiere mucho a nivel mundial. En la mayoría de países las investigaciones son incompletas, muy escasas, o casi nulas, en otros existen estudios parciales o publicados en forma dispersa, y solamente en algunos países desarrollados se han descrito y analizado en forma más o menos completa las desigualdades en salud.

III.5 El informe Black afirmó con claridad que los factores socioeconómicos del medio permitían explicar más adecuadamente la mayor parte de las desigualdades encontradas. Además, otros estudios mostraron cómo después de eliminar estadísticamente el impacto de distintos hábitos de riesgo, las desigualdades persistían en gran medida. Igualmente, el informe «The Health Divide» fue también taxativo al comentar, por un lado, que las desigualdades en salud entre los grupos sociales eran reales y no fruto de un error estadístico o de la teoría de la movilidad social, y por otro, que existían pruebas concluyentes de que los factores socioeconómicos eran los más importantes a tener en cuenta. 
III.6 Los estudios realizados en otros países, fruto del estímulo del informe Black, han mostrado la existencia de las desigualdades sociales en salud, que persisten e incluso aumentan en algunos de los países de la Unión Europea.

\section{CAPITULo IV}

IV.1 Aunque la cantidad y la calidad de las investigaciones sobre desigualdades en salud en España ha aumentado notablemente en los últimos años, la tradición en la investigación y los estudios por clase social, privación material u otros indicadores sociales de salud son aún escasos y las limitaciones en este campo de investigación son aún muchas.

IV.2 Las causas que explican esta situación son: 1) el corto desarrollo histórico de la salud pública en España fruto del vacío existente durante la dictadura franquista; 2) la escasa sensibilidad manifestada por los profesionales de la salud acerca de la relación existente entre los fenómenos sociales y la salud; 3) el aún limitado interés existente por el tema de las desigualdades en salud tanto en la comunidad científica como los medios de comunicación; 4) las limitaciones de las fuentes de información disponibles en nuestro país, lo cual se debe tanto a la falta de comparabilidad de las variables sociales en los sistemas de información sanitarios, como a la ausencia de variables sanitarias en otros sistemas de información en los que sí se dispone de variables sociales.

\section{Capítulo V}

V.1 El estudio ecológico en áreas pequeñas en España (zonas menores que la provincia) ha permitido mostrar la existencia de desigualdades sociales en mortalidad en los años 1990-1992 a nivel de áreas pequeñas, Comunidades Autónomas y regiones.

V.2 Las Comunidades Autónomas con un mayor porcentaje de desempleados son, sobre todo, las que poseen un alto nivel de actividad agrícola latifundista, como Andalucía o Extremadura, con tasas de desempleo próximas al 30 por 100, así como las que poseen un alto nivel de actividad industrial en crisis, como Euskadi y Asturias. Las Comunidades Autónomas con el mayor nivel de analfabetismo son aquellas que históricamente han tenido un menor nivel de renta y más población rural: Andalucía, Extremadura, Murcia o Castilla-La Mancha. Por ejemplo, Andalucía con menos del 18 por 100 de la población española concentra casi un tercio de los analfabetos existentes en nuestro país.

V.3 A partir del estudio realizado parecen dibujarse tres tipos de regiones: una «región alta», con mayores recursos situada en el norte del país, con Comunidades Autónomas como La Rioja, Cantabria, Aragón y Navarra, en las que predomina un elevado porcentaje de población que vive en zonas aventajadas (con un mayor nivel socioeconómico); una «región media», con un nivel intermedio de recursos, en la que se incluyen la Comunidad Valenciana y Galicia, que presentan un elevado número de habitantes y zonas de nivel medio y un pequeño número de zonas deprimidas (bajo nivel socioeconómico). Finalmente, una «región baja», con pocos recursos en las que se incluyen comunidades como Andalucía, Extremadura o Canarias, con un elevado porcentaje de población y zonas de nivel deprimido (bajo nivel socioeconómico). 
V.4 En lo que respecta a la mortalidad por todas las causas, y usando como instrumento comparativo la Razón de Mortalidad Comparativa (RMC) que compara la mortalidad estandarizada por edad de cada Comunidad Autónoma con la de España, destaca la mortalidad más alta en las comunidades situadas en la región de «nivel bajo» (Ceuta, Murcia, Andalucía y Canarias), Baleares (cuya mortalidad parece responder a causas distintas a las de otras comunidades '), así como la mejor situación de comunidades como Castilla y León, Navarra y Aragón. Por ello, este estudio confirma el patrón hallado en otros estudios presentados a nivel de las Comunidades Autónomas. Las Comunidades Autónomas del sur tienden a concentrar los mayores porcentajes de población viviendo en zonas con Razones de Mortalidad Comparativa mayores de 110 (superiores en un 10 por 100 a la del promedio de España), así como los mayores porcentajes en el número de zonas que se hallan en esa situación. Tal es el caso de Extremadura, Andalucía, Canarias y Murcia.

V.5 Se confirma en este estudio la desigualdad de mortalidad entre la España del norte-noreste (nivel alto) y la España del sur-suroeste (nivel bajo). En las regiones de nivel de vida alto el porcentaje de población que vive en zonas deprimidas, así como el porcentaje de población que vive en zonas con alta mortalidad son bajos. En cambio, en la región de nivel de vida bajo, más de la mitad de la población vive en zonas deprimidas y existe un elevado porcentaje que vive en zonas de alta mortalidad.

V.6 Se observa una asociación entre los indicadores sociales usados y la mortalidad. Así, tanto a nivel de Comunidades Autónomas como de áreas pequeñas, existe una asociación entre las variables de privación material y la mortalidad. Por un lado, todas las Comunidades Autónomas con menor nivel económico (mayor privación material), salvo Castilla-La Mancha, muestran niveles de Razón de Mortalidad Comparativa por todas las causas de muerte de más de 100 presentan un elevado porcentaje de población viviendo en zonas con Razón de Mortalidad Comparativa superior a 110.

V.7 El estudio de áreas pequeñas en España (zonas menores que la provincia) ha mostrado la asociación positiva en forma de gradiente entre los indicadores de privación material y la mortalidad por todas las causas.

El estudio realizado llega a conclusiones globales similares a las obtenidas por los estudios sobre desigualdades en mortalidad en España realizados anteriormente.

V.8 En relación al estado de salud percibido, se observa en ambas encuestas de 1987 y 1993 cómo el porcentaje de hombres y mujeres con salud deficiente (estado de salud regular, malo o muy malo) aumenta a medida que disminuyen los recursos de la clase social de los individuos; la desigualdad también aumenta a medida que lo hace la edad.

V.9 Entre 1987 y 1993 se ha producido una disminución de la desigualdad en la valoración del estado de salud entre las clases sociales, particularmente para las edades a partir de los cincuenta y cinco años. Sin embargo, esta disminución de la desigualdad se ha producido particularmente en las Comunidades Autónomas con renta familiar disponible igual o mayor a la renta media nacional. En las Comunidades Autónomas con renta familiar disponible significativamente inferior a la media nacional las desigualdades entre las clases sociales han aumentado.

1 No ha sido objeto de este estudio el hacer un análisis de las causas de mortalidad. De ahí que no haya sido posible hacer un estudio detallado del caso particular de Baleares. 
DERECHOS Y LIBERTADES

V.10 Existen evidencias de que una serie de padecimientos crónicos, tales como trastomos respiratorios (asma y/o bronquitis crónica), tensión arterial elevada y diabetes, son padecidas con mayor frecuencia a medida que descendemos en la clase social de los individuos. Esta tendencia no es homogénea para hombres y mujeres; así, estas desigualdades se constatan en la tensión arterial y la diabetes para las mujeres, y en los transtornos respiratorios para los hombres. Una enfermedad crónica, las alergias, ha sido hallada con mayor frecuencia en las clases más aventajadas, a diferencia de las otras enfermedades.

V.11 De 1987 a 1993 ha habido un descenso en la prevalencia de tabaquismo entre los hombres, aumentando ligeramente entre las mujeres. Entre los hombres, la prevalencia de tabaquismo es mayor conforme disminuye la clase social. En mujeres, la desigualdad es inversa, es decir, la prevalencia es mayor en las clases con más recursos. Tanto en los hombres como en las mujeres las desigualdades son más evidentes en las regiones de mayor renta aun y cuando la prevalencia de fumadores es menor. En hombres, las desigualdades sociales no parecen haber aumentado significativamente de 1987 a 1993. En mujeres, sin embargo, la diferencia de todas las clases con respecto a la clase I ha disminuido de 1987 a 1993. Dado que en 1987 es el momento en el que la clase I tiene una probabilidad mayor de fumar, esta evolución es claramente negativa.

V.12 De 1987 a 1993 ha habido un descenso en la prevalencia de alcoholismo. En hombres, la prevalencia de quienes beben alcohol en exceso aumenta conforme disminuyó la clase social. En mujeres, en cambio, no se observa un patrón claro. El consumo de alcohol es mayor en las regiones de menor renta entre los hombres, y en las mujeres lo es en las Comunidades Autónomas de mayor renta. Además, en los varones las desigualdades han aumentado entre 1987 y 1993.

V.13 De 1987 a 1993 ha habido un aumento en la proporción de personas que hacen ejercicio físico. En cuanto al ejercicio físico durante el tiempo libre, existe una clara relación entre clase social y sedentarismo. Tanto en hombres como en mujeres a medida que disminuye la clase social aumenta el porcentaje de personas que no practican ejercicio físico. Este porcentaje además es mayor en las Comunidades Autónomas con renta familiar más baja. Asimismo, las desigualdades entre las clases relativas a la práctica de ejercicio físico han aumentado del 87 al 93.

V.14 En 1993 se ha producido, en comparación a 1987, un aumento de las consultas al médico en hombres y en mujeres ${ }^{2}$. Sin embargo, cuando se considera tan sólo a las personas con salud deficiente, las consultas al médico han disminuido en mujeres y han aumentado en hombres del 87 al 93.

V.15 En la consulta al médico no parecen existir claras evidencias de conductas diferentes por parte de las distintas clases sociales. $\mathrm{El}$ análisis sugiere la existencia de cierta desigualdad en las mujeres en 1993 entre la clase 1, que consultaría más, y las clases II, III, y IV, clases que lo harían menos. Sin embargo, en conjunto hay una ausencia de desigualdades en la consulta al médico. Más de un 80 por 100 de las personas que han consultado al médico lo han hecho en un centro sani-

2 Esta diferencia podría deberse a que las encuestas se realizaron en distintos períodos del año. 
tario público, en comparación con las consultas en entidades privadas o sociedades médica. Esta proporción es claramente muy diferente según la clase social de los encuestados. Así, en ambas encuestas, alrededor de un 60 por 100 de la clase I, más del 70 por 100 de la clase II, y alrededor de un 90 por 100 de las clases III y IV han acudido al médico en un centro sanitario público. En conjunto, más de un $\mathbf{8 0}$ por 100 de las personas que han consultado al médico lo han hecho en un centro sanitario público, en comparación a haberlo hecho en una consulta privada o sociedad médica.

V.16 En 1993 hubo un aumento de las tasas de hospitalización en comparación con 1987. El análisis de las tasas de hospitalización no revela, sin embargo, tendencias claras que indiquen patrones de desigualdad. No se observan diferencias entre las clases sociales, como tampoco aparecen de forma clara entre las Comunidades Autónomas de acuerdo al nivel de renta de las mismas.

V.17 En nuestro país existe un claro patrón de desigualdad en la utilización de servicios dentales en 1987 y en 1993. La proporción de personas que acuden al dentista disminuye a medida que descendemos en la escala social. Pero además, esta desigualdad entre las clases parece haber aumentado de 1987 a 1993. En hombres este aumento ha sido más marcado en las Comunidades Autónomas con renta más baja. En mujeres, por el contrario, en las Comunidades Autónomas con mayor renta es donde el aumento de la desigualdad es más nítido. Parece claro que la financiación eminentemente privada de los servicios dentales está generando desigualdades sociales que no se presentan cuando la financiación es pública.

\section{Capitulo VI}

VI.1 El objetivo primordial de la investigación sobre desigualdades en salud se ha centrado en el conocimiento de la existencia, la magnitud, la evolución, las causas y las consecuencias de las desigualdades en salud de las poblaciones.

VI.2 La experiencia histórica demuestra que el simple hecho de ignorar la existencia de iniquidades no hará que éstas desaparezcan, se autolimiten o reduzcan, ya que las políticas económicas y sociales que dejan al mercado el libre albedrío de la distribución de recursos crean un aumento de las desigualdades.

VI.3 La mayor parte de investigaciones sobre desigualdades sociales en salud coinciden en señalar que son las causas socioeconómicas y políticas de carácter estructural las que mejor explican las desigualdades existentes. Estas causas no son inevitables, sino que en muchos casos pueden modificarse con un coste social razonable, o en todo caso, forman parte del conjunto de opciones que la sociedad debe elegir.

Las intervenciones destinadas a disminuir las desigualdades deben dar respuesta a cuatro preguntas generales: ¿qué debe hacerse?, ¿sobre quién?, ¿dónde aplicarlas? y, finalmente, ¿quién debe aplicarlas? Así, es necesario caracterizar varios aspectos: en primer lugar, el tipo de intervencion, ya sea de tipo socioeconomico o sanitario; segundo, la población sobre la que se debe actuar, ya sea sobre la población general o sobre grupos de riesgo vulnerables; y finalmente, el nivel geográfico sobre el que intervenir: nacional, regional o local. 
VI.4 El objetivo de las políticas no puede ser eliminar las desigualdades en salud, sino reducir esas desigualdades al mínimo, creando oportunidades iguales y reduciendo o eliminando aquellos factores que se vean como evitables e injustos. Para ello, las intervenciones generales deben concretarse, en cada caso, en acciones prioritarias específicas, racionales y solidarias, relacionadas con los factores causales de desigualdades más relevantes y modificables. Así, primero, se debe analizar cómo reducir las desigualdades sociales más importantes y de solución más factible, y en segundo lugar, poner en práctica las políticas más eficientes. Para lo primero se requiere información y análisis, para lo segundo, se necesita plantear y resolver conflictos y combatir las resistencias políticas a una estrategia pro equidad.

Estas políticas debieran realizarse tanto a nivel estatal como autonómico y municipal, con el fin de reducir no sólo las desigualdades entre las distintas regiones autonómicas, sino también intraautonómicas y municipales, con la finalidad de que los gobiernos tanto regionales como municipales se sientan implicados en la lucha contra la desigualdad.

VI.5 Los siete principios generales que las políticas sanitarias de reducción de las desigualdades deben tener en cuenta son:

a) La mejora de las condiciones de vida y trabajo de la población. La intervención sobre el acceso y calidad de los alimentos, el agua potable, o la contaminación ambiental son ejemplos de acciones generales sobre la población. Las políticas ante el desempleo o la pobreza son ejemplos de acciones específicas.

b) La necesidad de lograr que se adopten estilos de vida más saludables, en especial en los grupos más vulnerables y necesitados.

c) La descentralización del poder y la toma de decisiones con el fin de promover la participación popular acorde con las propias necesidades de la población. Ello implica, que la población más vulnerable halle la manera de expresar sus necesidades.

d) La valoración del impacto sobre la salud en el campo de la accion intersectorial. Ello conlleva el que se tenga en cuenta el objetivo de mejorar la salud en cualquier plan de desarrollo social o económico.

e) Dada la estrecha interpelación entre las políticas sociales y sanitarias de los países debe existir un interés mutuo a nivel internacional para controlar diversos problemas de salud. La producción y exportación de productos tóxicos, de fármacos no autorizados, los flujos migratorios, o el control de la contaminación ambiental son ejemplos de esa interrelación.

f) Lograr el máximo acceso, y la mayor calidad de la atención sanitaria para toda la población. Se trata de, por ejemplo, examinar la distribución de recursos según las necesidades sociales y sanitarias, la distribución geográfica de los servicios o las razones de por qué no se usan servicios esenciales.

g) Realizar la investigación, seguimiento y evaluación adecuados sobre las políticas para conocer su efectividad real.

VI.6 Entre las estrategias más importantes citadas en la literatura especializada destacan las siguientes: primero, actuar sobre las desigualdades en salud de los niños porque son acciones relevantes, efectivas y son consideradas situaciones inaceptables para el público; segundo, mejorar las condiciones laborales y de vivienda de la 
población, y muy en especial la de los grupos más necesitados; tercero, actuar sobre áreas geográficas con privación material y social muy alta; cuarto, lograr que se adopten hábitos de conducta más saludables, en especial en los grupos más vulnerables; y quinto, mejorar el acceso y la calidad de la atención sanitaria de toda la población.

VI.7 El informe concluye mostrando la experiencia positiva de reducir las desigualdades sociales en la Comunidad Autonómica Vasca y en el municipio de Barcelona, experiencias que han sido impulsadas por autoridades que pertenecen a distintas opciones políticas pero que comparten la misma sensibilidad social. La reducción de desigualdades sociales debiera ser el proyecto de todas las fuerzas políticas y sociales democráticas.

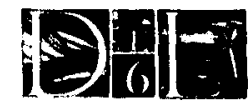

